\title{
Heteronormative Biopolitik und die Verhinderung von trans Schwangerschaften'
}

Annika Spahn

\section{Einleitung}

\begin{abstract}
»[...] a deviation from what is known and expected. That has been the story of my entire pregnancy - no one has known quite what to make of it, or been able to truly understand what it means. $\aleph^{2}$ (Beatie 2008:3)
\end{abstract}

Soweit Thomas Beatie in der Einleitung seiner Autobiographie. Beatie, der bei seiner Transition seinen Uterus und seine Eierstöcke behielt (wie viele andere trans Männer auch), war nach eigener Aussage 2008 weltweit der erste Mann, der ein Kind zur Welt brachte (ebd.:6; 309f.). Unter anderem J. Jack Halberstam widerspricht dem deutlich (Halberstam 2012:31f.).

Beaties Geschichte erlangte weltweit Aufmerksamkeit (ebd.:263ff.) und prägt bis heute das Narrativ um schwangere trans Männer. Seine Frau konnte nach einer Hysterektomie nicht mehr schwanger werden und eine Leihmutterschaft ergab keinen Sinn für das Paar, da er selbst einen Uterus hatte. Deswegen entschied sich das Paar, dass er schwanger werden sollte. Beaties Schwangerschaft machte das erste Mal öffentlich darauf aufmerksam, dass

1 Ich danke Maike Kemper, Ravna Siever, Sasha, @engelsesser, @mensch.dieser.blog und allen anderen Aktivist_innen, die an meiner Studie teilgenommen haben oder mir wichtiges Feedback gaben für ihre Unterstützung und Hilfe.

2 Übersetzt: »[...] eine Abweichung von dem, was bekannt ist und erwartet wird. Das war die Ceschichte meiner gesamten Schwangerschaft - niemand hat gewusst, wie sie zu verstehen ist oder was sie genau bedeutet.«[AS] 
auch (manche) trans Männer schwanger werden wollen und können. Gleichzeitig zeigte sein Fall auch, mit wie viel Hass und Gewaltandrohungen eine solche Überschreitung von Geschlechterrollen im Sozialgefüge geahndet wird (Beatie 2008:250) und mit welchen Diskriminierungen, insbesondere im medizinischen und juristischen System, eine Schwangerschaft einer nicht-cis-weiblichen Person verbunden ist (ebd.:199).

In diesem Beitrag möchte ich ein Augenmerk auf diese spezifische Diskriminierung in Medizin und Justiz legen von Schwangeren, die keine cis Frauen sind, und argumentieren, dass Schwangerschaften von trans Männern und nicht-binären Personen mit Uteri aktiv von Staat, Gesellschaft und Medizin verhindert werden. Dabei liegt mein Augenmerk auf trans Männern; vieles ist aber auch auf nicht-binäre Personen übertragbar, sofern sie einen Uterus haben. ${ }^{3}$ Unter strans Schwangerschaften alle Schwangerschaften von Personen, deren bei der Geburt zugewiesenes Geschlecht (in diesem Falle meistens weiblich) nicht, nicht immer oder nur teilweise mit deren selbstbestimmten Geschlecht übereinstimmt. ${ }^{4}$

Zunächst soll ein Überblick über den Forschungsstand zu trans Schwangerschaften dargelegt werden. Dies geschieht insbesondere im Hinblick darauf, in welchen wissenschaftlichen Disziplinen ein Interesse und eine Sensibilität für trans Schwangerschaften erkennbar ist. Anschließend werden knapp die juristischen, medizinischen und gesellschaftlichen Rahmenbe-

3 Die in diesem Artikel zitierten Interviewpassagen stammen aus einer Studie, die im Rahmen meiner M.A.-Arbeit zu Schwangerschaft bei trans Männern und Uterustransplantationen für trans Frauen durchgeführt wurde. Im Rahmen dieser Studie wurden ca. 50 trans Personen aus Deutschland mithilfe eines Cruppendiskussionsverfahren befragt. Darunter war ein Freundeskreis dreier trans Männer, eine trans Selbsthilfegruppe, in der vor allem trans Frauen organisiert waren, und eine Selbsthilfegruppe für trans Männer und ihre Angehörigen. Die Gruppendiskussionen wurden transkribiert und mit Kruses integrativem Basisverfahren ausgewertet. Insgesamt handelte es sich um eine explorative Forschung, das heißt die Zuspitzung auf die heteronormativ-biopolitische Verhinderung von trans Schwangerschaften ergab sich aus dem Material - als Forschungsfrage stand zu Beginn der Forschung nur, welche Einstellungen und politischen Forderungen trans Personen zu Schwangerschaft hatten (Spahn 2017).

4 Durch mein persönliches Umfeld, durch meinen queeren Aktivismus und durch meine wissenschaftliche Arbeit bin ich gut mit dem Themenkomplex trans vertraut. Das heißt: Ich interessiere mich für die Lebenssituation von trans Personen und deren Verbesserung und versuche mich selbst ständig dazu weiterzubilden, welche Forderungen und Diskurse es in der trans Community gibt. Als nicht-transgeschlechtliche Person versuche ich mir meine eigene Privilegierung vor Augen zu führen und zu reflektieren. 
dingungen dargestellt, in die trans Schwangerschaften in Deutschland eingebettet sind. Im Hauptteil wird aufbauend auf eine Studie und die juristischen, medizinischen und sozialen Gegebenheiten skizziert, inwiefern trans Schwangerschaften in Deutschland verhindert werden. Für die Analyse wird sich auf Michel Foucaults Konzept der Biomacht gestützt. Im Fazit werden die wichtigsten Ergebnisse dargelegt und, angelehnt an Ute Kalender, diskutiert, inwiefern es sich hier um eine Form von neuer Eugenik handelt.

\section{Der Forschungsstand}

Während eine sozialwissenschaftliche Beschäftigung mit trans Schwangerschaften weitestgehend noch aussteht (De Sutter 2014; Halberstam 2012) wird das Thema in journalistischen (Duhaime-Ross 2016; Gordon 2015; Grady 2015; Hempel 2016; Hening 2014; Prantl 2011; Queerulant_in 2015) und aktivistischen (Beatie 2008; Diamond 2011; Janssen 2016; Richards 2016; Wallace 2010) Kontexten bereits breiter verhandelt. In der Soziologie der Geburt (Villa/Moebius/Thiessen 2011) und der Soziologie der Schwangerschaft (Hirschauer 2014) fehlen schwangere nicht-weibliche Personen gänzlich. Eine medienwissenschaftliche Analyse über trans Schwangerschaften findet sich bei Verlinden (2012). Außerdem lässt sich ein langsam wachsendes Interesse in der US-amerikanischen Medizin erkennen. Hierbei werden folgende Themen verhandelt: die Rolle von Testosteron vor und während der Schwangerschaft, Schwangerschaftskomplikationen und psychische Probleme aufgrund sozialer Normen während der Schwangerschaft sowie Stillen und Reproduktionsmöglichkeiten (Obedin-Maliver/Makadon 2016). Als wichtiges Thema werden außerdem die Unsicherheiten und diskriminierenden Handlungen und Äußerungen von medizinischem Personal genannt (Obedin-Maliver 2015). Auch im Deutschsprachigen findet sich mittlerweile ein Artikel zum Kinderwunsch von trans Personen (Flütsch 2017). Zusammenfassend gibt es eine erste medizinische Beschäftigung mit schwangeren trans Personen und Empfehlungen für den respektvollen gynäkologischen Umgang mit ihnen - allerdings, so meine These, sind diese den meisten Gynäkolog_innen nicht bekannt. 


\section{Medizinische, rechtliche und gesellschaftliche Kontexte von trans Schwangerschaften in Deutschland}

Trans Männer können, ebenso wie alle anderen Menschen, ein Bedürfnis nach Schwangerschaft und Kindern haben (Hempel 2016: Absatz 4; Wierkx u.a. 2012). Eine virilisierende, das heißt vermännlichende, Hormontherapie bei trans Männern führt nur zu einer reversiblen Amenorrhoe (dem Ausbleiben der Menstruation). Es besteht vor einer Adnektomie (der Entfernung der Eierstöcke) und Hysterektomie (der Entfernung des Uterus) also jederzeit durch Absetzen der virilisierenden Hormone die Möglichkeit einer Schwangerschaft (De Sutter 2014:217ff.). Hierbei ist anzumerken, dass Testosteroneinnahme keine sichere Verhütungsmethode darstellt und einige trans Personen auch mit der Einnahme von Testosteron, sofern sie ihren Uterus behalten, menstruieren und schwanger werden können. Viele trans Männer sind an reproduktiven Fragen interessiert und frieren vor einer Testosteron-Behandlung bzw. einer Ovarektomie Eizellen ein (Hempel 2016, Absatz 30). ${ }^{5}$ Genauso, wie medizinische Faktoren einen Einfluss auf trans Schwangerschaften haben, haben dies auch juristische Diskurse:

2011 urteilte das Bundesverfassungsgericht, dass Teile (der Sterilisationszwang und der Zwang zu geschlechtsangleichenden Maßnahmen) des Transsexuellengesetzes (TSG) verfassungswidrig sind (BVerfG 2011 und Schirmer 2012:244). Damit wurden zwar bei weitem keine juristischen und gesellschaftlichen Normen über Schwangerschaft und Geschlechter- und Körperbilder aufgeweicht, dennoch hatte dies zur Folge, dass in Deutschland nun Menschen mit juristisch männlichem Personenstand Kinder gebären können ${ }^{6}$. Wenn allerdings eine Person mehr als 300 Tage nach der Änderung des Vornamens im Rahmen des TSG ein Kind bekommt, wird die Änderung des Vornamens hinfällig, da dann davon ausgegangen wird, dass die entsprechende Person sich doch dem weiblichen Geschlecht zu-

5 Dies stellt allerdings für viele trans Männer keine ideale Lösung dar. Erstens ist bis heute unklar, wie erfolgreich die Kryokonservierung von Eizellen ist, das heißt wie gut die Chancen darauf sind, mit diesen Eizellen später schwanger werden zu können. Zweitens kann mit diesen Eizellen nur die Person schwanger werden, der sie auch entnommen wurden, da in Deutschland Eizellspenden illegal sind. Drittens ist der Prozess der Eizellentnahme mit sehr viel Aufwand und potentiellen Triggern für Dysphorie verbunden, z.B. der Abbruch der Hormonersatztherapie und invasive gynäkologische Untersuchungen (Toze 2018:203).

6 Für inter Personen war dies schon vorher theoretisch möglich. 
gehörig fühlt. Dies kann zwar wieder revidiert werden, wenn schwerwiegende Gründe vorliegen (Adamietz 2011:135ff. und \$7 TSG), stellt jedoch für trans Personen mit Kinderwunsch eine große Hürde dar. Hat eine Person ihren Personenstand und ihren Vornamen geändert, werden diese nach der Geburt eines Kindes nicht rückgängig gemacht. Außerdem werden trans Personen auch nach einer Personenstands- und Vornamensänderung über das TSG mit ihrem Deadname und ihrem zugewiesenen Geschlecht in die Geburtsurkunde des Kindes und in das Geburtenregisters eingetragen. Das bedeutet: Ein gebärender trans Mann ist juristisch gesehen immer die "Mutter« des Kindes. Es ist außerdem davon auszugehen, dass sich juristisches Fachpersonal, das nicht für trans Themen sensibilisiert ist, gegenüber schwangeren trans Personen diskriminierend verhält, einer schwangeren trans Person ihr Geschlecht aberkennt oder eine Personenstandsänderung erschwert oder verwehrt - schließlich ist eine Personenstands- und Vornamensänderung in Deutschland eine richterliche Entscheidung und wird nicht durch die Vorlage der geforderten Gutachten garantiert.

Trans Personen erfahren in der Gesellschaft massive Diskriminierungen, die sich insbesondere auf heteronormative Rollenerwartungen beziehen. Dies sind beispielsweise Mikroaggressionen, Ausgrenzung innerhalb Peergroups bei ihrem Coming-Out sowie körperliche Gewalt in öffentlichen und privaten Räumen. Als Resultat dieser transfeindlichen Strukturen sind Depressionen, suizidale Handlungen und Substanzmissbrauch unter trans Personen weit verbreitet (Cerwenka/Nieder 2015:19; Nordmarken 2014:129ff.). Eine Studie der Agentur der Europäischen Union für Grundrechte (FRA) zeigte unter anderem, dass in den zwölf Monaten vor der Studie $54 \%$ der befragten trans Personen aufgrund ihrer Transgeschlechtlichkeit diskriminiert oder belästigt wurden (FRA 2014: 2). Jede zweite befragte Person erlebt etwa einen Gewaltvorfall pro Jahr; 34 \% geben an, innerhalb des letzten Jahres körperliche oder sexualisierte Gewalt erfahren oder angedroht bekommen zu haben (ebd.).

Die Körper von trans Personen werden von der Mehrheit als anti-normativ gelesen. Sie erleben aufgrund dessen Diskriminierungen und physische, emotionale, institutionelle, verbale und sexualisierte Gewalt - sie werden also, um es mit Popitz Worten (2010:61ff.) auszudrücken, sanktioniert, weil sie nicht in verbindliche gesellschaftliche Normvorstellungen von Geschlecht und Körper passen. Wie oben beschrieben, sind juristische Normen darüber, wie vergeschlechtliche Körper auszusehen haben, seit 2011 in Bezug 
auf trans aufgeweicht worden - dies gilt aber nicht für die etwas schwerer zu fassenden sittlichen bzw. moralischen Normen (Schimank 2002:40). Allerdings gehe ich aufgrund der vorherrschenden Gewalt und sozialen Diskriminierung davon aus, dass es sozial gesehen nicht egal ist, wie vergeschlechtlichte Körper auszusehen haben.

David Valentine schreibt in der Einleitung seiner Ethnographie Imagening Transgender: »I want to argue that stransgender, rather than being an index of marginality [...] is in fact a central site where meaning about gender and sexuality are being worked out.« (Valentine 2007:14) ${ }^{7}$ Trans wird hier also zu einer Kategorie, anhand der sich Verschiebungen im aktuellen Geschlechter- und Sexualitätsverhältnis und juristische, biopolitische, medizinische und psychologische Regulierungen von Geschlecht nachzeichnen lassen (auch in Schirmer 2012:246). An dieser Stelle ist es interessant zu fragen, ob dies auch für das Thema Schwangerschaft gilt, das eigentlich - so meine These - gesellschaftlich ausschließlich mit Weiblichkeit gleichgesetzt wird.

\section{Wie trans Schwangerschaften verhindert werden}

Trans Personen erleben in medizinischen Kontexten strukturelle und direkte, persönliche Diskriminierung sowie Gewalt durch Pathologisierung, Objektifizierung, Abhängigkeiten, Entmündigung und vieles mehr (Pohlkamp 2014:281ff.). Um juristisch und medizinisch transitionieren zu können, (das heißt die Änderung von Vornamen und Personenstand sowie Hormonersatztherapien, ggf. pubertätsblockierende Medikamente und/oder geschlechtsangleichende Operationen durchführen zu können,) müssen sich trans Personen einer psychiatrischen Diagnose unterwerfen ${ }^{8}$ und eine begleitende Psychotherapie absolvieren. Sie müssen dabei in medizinischen Settings immer wieder heteronormativen Geschlechtervorgaben entsprechen, um in ihrem tatsächlichen Geschlecht anerkannt zu werden.

Medizinisch werden trans Schwangerschaften schon alleine dadurch aktiv verhindert, dass trans Männern oftmals beim Beginn einer Hormon-

7 Übersetzung: »lch möchte argumentieren, dass «transgender « nicht so sehr ein Index von Marginalisierung ist als [...] ein zentraler Raum, in dem Bedeutungen von Ceschlecht und Sexualität ausgehandelt werden.«[AS]

8 Dies wird sich mit der Einführung des ICD 112022 ändern, da trans dann nicht mehr als "Störung der Ceschlechtsidentität« gilt, sondern als Geschlechtsinkongruenz (Kraus 2018). 
ersatztherapie geraten wird, eine Hysterektomie und eine Adnektomie durchführen zu lassen. Ohne Uterus und Eizellen ist eine eigene Schwangerschaft fast ausgeschlossen. Begründet wird der Rat zur Entfernung von Uterus und Eierstöcken mit einem steigenden Krebsrisiko bei der Einnahme von Testosteron - allerdings lässt sich dieses wissenschaftlich nicht nachweisen (Toze 2018:197) ${ }^{9}$. In vielen Fällen wird eine Hysterektomie nicht aus medizinischen Gründen durchgeführt, sondern um die Dysphorie der trans Person zu lindern. Eine Studie konnte allerdings zeigen, dass der meistgenannte Grund für eine Hysterektomie unter trans Männern präventive Gesundheitsversorgung war (Rachlin u.a. 2010, zitiert nach Toze 2018:199). Außerdem werden trans Männer vor dem Start einer Hormonersatztherapie $\mathrm{zu}$ selten und zu schlecht über reproduktive Fragen (z.B. über die Möglichkeit, Eizellen einfrieren zu lassen) aufgeklärt - womit ebenfalls spätere eigene Schwangerschaften verhindert werden (Wierckx 2012).

Trans Männer, die gerne vaginalen, empfangenden, penetrativen Sex haben, werden beispielsweise häufig von Ärzt_innen nicht als Männer anerkannt - dasselbe gilt, wenn Ärzt_innen über einen trans Mann herausfinden, dass er (irgendwann) schwanger werden möchte. Diese weiblich konnotierten Wünsche und Vorlieben werden als Kontraindikation zu einer psychiatrischen trans Diagnose angesehen, die für eine rechtliche und medizinische Transition benötigt wird. Das bedeutet, dass einem trans Mann ggf. eine (lebensrettende) Transition oder sogar eine nicht mit der Transition zusammenhängende medizinische Behandlung verweigert wird, weil er schwanger werden möchte (Verlinden 2012:115; Toze 2018:202). Es ist außerdem zu erwarten, dass im Falle einer ungeplanten Schwangerschaft eines trans Mannes medizinisches Personal, das nicht zu trans Themen fortgebildet wurde, den trans Mann zu einem Abbruch dieser Schwangerschaft drängen wird, um ein cis-normatives Geschlechterbild aufrecht zu erhalten. Ebenso kann davon ausgegangen werden, dass sich Gewalt in der Geburtshilfe (Mundlos 2015) potenziert, wenn die gebärende Person keine cis Frau ist. Dabei handelt es sich vermutlich um zusätzliche, andere Formen von Ge-

9 Davon abgesehen treten beispielsweise Endometriumkarzinome, das heißt Krebs der Uterusschleimhaut, in den allermeisten Fällen nur bei Personen über 65 Jahren auf, während die Hysterektormien bei trans Männern meistens im Alter von 20-30 Jahren durchgeführt werden. Die Betroffenen hätten also ca. 40 Jahre Bedenkzeit für eine Hysterektomie, wenn diese aus präventiven Gründen erfolgen sollte (ebd.: 198). 
walt im Vergleich zu cis Frauen, also dass zum Beispiel der selbstgewählte Name, die Bezeichnung »Vater« o.ä., die richtigen Pronomen etc. nicht respektiert werden und medizinisches Personal in einer solchen Situation überfordert bis ablehnend reagiert. Zusätzlich ist zu erwarten, dass ungeplante und den Patient_innen unbewusste Schwangerschaften von medizinischem Personal bei Menschen mit männlichem Personenstand nicht erkannt werden, was z.B. bei einer Eileiterschwangerschaft oder einer Röntgenuntersuchung gefährlich sein kann.

Auch in den Gruppendiskussionen zu trans und Schwangerschaft mit trans-aktivistischen Gruppen ließ sich immer wieder die Angst vor Diskriminierung im medizinischen Sektor feststellen, die gemeinsam mit anderen Faktoren dazu beiträgt, dass trans Personen sich gegen eine eigene Schwangerschaft entscheiden (Spahn 2017:34ff.). Außerdem zeigt sich eine Angst vor juristischen Diskriminierungen, also dem Rückgängigmachen einer Vornamensänderung durch die Geburt eines Kindes und die falsche Eintragung in die Geburtsurkunde des Kindes. Letzteres wurde 2018 vom Bundesverfassungsgericht aufrechterhalten (Göbel 2018). Eine betroffene Person muss aktiv dafür kämpfen, den eigenen Namen doch behalten zu dürfen, und ist in diesem Prozess ebenfalls Diskriminierungen und insbesondere dem Absprechen der eigenen Identität ausgesetzt. Die Teilnehmenden an der Studie erleben den Staat in Hinblick auf diese Regelungen als bürokratisch, undurchsichtig und diskriminierend (ebd.:34ff.).

\section{Feminisierung von Schwangerschaft und internalisierte Cis-Normativität}

Im Fall von Thomas Beatie zeigt sich die Wirkmächtigkeit dessen, was als `soziale Feminisierung von Schwangerschaft bezeichnet werden kann: Beatie (und anderen trans männlichen und nicht-binären Personen, die schwanger werden,) wird ihr selbstbestimmtes Geschlecht abgesprochen - es heißt, sie könnten keine srichtigen` Männer (bzw. nicht-binäre Personen) sein, da sie sich für eine Schwangerschaft entscheiden. Diese Stimmen kommen dabei nicht nur von transfeindlichen cis Personen, sondern auch von innerhalb der trans Community (Verlinden 2012:114). In trans-aktivistischen Gruppen wird über das Thema Schwangerschaft kaum diskutiert (Janssen 2014). Toze (2018) merkt an, dass es sich bei dieser Ablehnung schwangerer trans Män- 
ner innerhalb der trans Community nicht nur um internalisierte Cis-Normativitä $\mathrm{t}^{10}$ handle, sondern auch um die Angst, die nur schwierig erreichte soziale Anerkennung von trans Personen zu verlieren: »How can anyone take us seriously as men if some of us get pregnant?« (Toze 2018:204)

Gesellschaftlich wird Geschlecht eben an vermeintlichen Körpermerkmalen festgemacht (da diese aufgrund von Bartwuchs, Körperfettverteilung etc. vermutet und zugeschrieben werden), nicht an der Selbstaussage einer Person oder anderen Faktoren. Die Schwangerschaft einer trans Person zeigt somit die Grenze der sozial erlaubten geschlechtlichen Selbstbestimmung auf, die sich Aktivist_innen hart erkämpfen mussten und müssen - Organe, Hormonstatus und die Fähigkeit, schwanger zu sein und zu gebären, werden somit wieder zu Merkmalen, an denen das vermeintliche, cis-normative Geschlecht einer Person festgemacht werden kann. In der medialen Berichterstattung werden die Körper schwangerer trans Männer verbal entkleidet und seziert, um das zugewiesene Geschlecht buchstäblich $\mathrm{zu}$ enthüllen (Janssen 2014).

Auch in den Gruppendiskussionen war dies zu beobachten. Für eine der Gruppen ist Teilnehmer Gustav ${ }^{11}$, der als einziger trans Mann im Raum vor seinem Coming-Out und seiner Transition schwanger war, nicht als schwangerer trans Mann zu werten, denn er war damals nach Aussage eines Teilnehmenden »komplett noch Frau « ${ }^{12}-$ und schwangere trans Männer sind nach Meinung der Gruppe nur die, die auch als heteronormativ-männlich passen $^{13}$ (»Kai: du siehst nach außen hin männlich aus und bist schwanger«). Hierbei wird also eine Kategorisierung schwangerer trans Männlichkeit aufgemacht: Wer, wie Gustav, »Erik: noch in der weiblichen Hülle« ist, das heißt keine Transitionsmaßnahmen ergriffen hat, und schwanger wird, wird von der Gruppe akzeptiert.

10 Internalisierte Cis-Normativität bedeutet, dass eine trans Person, die in einer cis-normativen Gesellschaft aufgewachsen ist, diese Normen so verinnerlicht hat, dass er_sie sie gegen sich selbst verwendet.

11 Alle Namen anonymisiert.

12 Anders als üblich sind diese Zitate von mir bearbeitet, so dass der Dialekt der sprechenden Personen nicht erkennbar ist. Dies dient der Anonymisierung der Teilnehmenden.

13 »The work of achieving and making secure their rights to live in the elected sex status while providing for the possibility of detection and ruin carried out within the socially structured conditions in which this work occurred I shall call `passing«.«(Garfinkel 1967:118) 
»Erik: aber die Vorstellung halt - ich habe schon meine Hormone [die musst du dann absetzen] - ich hab vielleicht schon eine Brust-OP machen lassen setze dann die Hormone ab - weil ich hab noch meine Eierstöcke - Gebärmutter und werde jetzt schwanger - das ist was für mich etwas ähm [schizophrenes] [...] ja nee ähm das ist für mich soo - ähm - von jedem Kuchen will ich mir nur das Beste - also nicht das Beste aber - will ich mir etwas rausziehen - alsoo [ja] [mhm] - da hätte ich jetzt so meine Probleme - nicht wenn jemand noch in der w - weiblichen Hülle steckt und sagt - sich möchte schwanger werden< - da hätte ich jetzt weniger Probleme - als - oder gar kein Problem - aber - aber dieses soo - aber ich bin noch - schon Mann - mit äh paar OPs und dann noch schwanger werden des - ich weiß auch nicht.«

Wenn aber ein trans Mann, der bereits Transitionsmaßnahmen ergriffen hat, schwanger werden möchte, passt dies in den Augen der Gruppe nicht zueinander. Außerdem nehme man sich das Beste von jedem Kuchen - wird hier also als gierig und unzufrieden mit dem, was man hat, dargestellt. Ich vermute, diese Ablehnung eines schwangeren trans Manns während oder nach der Transition hängt auch mit heteronormativen Geschlechter- und Körperbildern zusammen. Männlichkeit und Schwangerschaft werden damit als sich ausschließende Konzepte angesehen. Kai spricht später darüber, dass er gerne Kinder gehabt hätte und sich Vorwürfe macht, nicht vor seiner Transition schwanger geworden zu sein. Auch hier wird die Möglichkeit einer Schwangerschaft nach oder während einer Transition ausgeschlossen. Anders sieht es für Kai aus, wenn er nun eine Frau kennenlernen würde, die bereits schwanger wäre - dann wäre er »Papa pur« und »mit schwanger wahrscheinlich«. Diese Anspielung auf das Couvade-Syndrom, bei dem cis Männer während einer Schwangerschaft ihres_ihrer Partner_in ebenfalls schwangerschaftsähnliche Symptome zeigen (Retzbach 2014) normalisiert hier den Wunsch nach einer männlichen Schwangerschaft. Das Couvade-Syndrom ist mit cis Männlichkeit verknüpft und eben nicht mit einem eigenen Uterus oder einer eigenen Schwangerschaft.

Schwangerschaft wird in allen Gruppendiskussionen immer wieder mit Weiblichkeit gleichgesetzt und grundsätzlich negativ bewertet - und doch, je länger die Gruppe über das Thema Schwangerschaft reflektiert, desto öfter referiert Kai darauf, dass er sich eine Schwangerschaft wünscht: 
»Kai: Ich wusste auch immer, dass ich irgendwo Mann bin - ja - aber trotz alledem hatte ich, auch wenn es vielleicht total widersprüchlich ist - und total mit dem Ganzen überhaupt nicht konform geht - äh - eine gewisse Zeit wo ich gerne schwanger geworden wäre-auch wenn es irgendwo total nicht passt-ja?«

Dies ist aber mit seinen persönlichen Überzeugungen, das heißt mit seinem Bild von Geschlecht, nicht vereinbar, denn seine erste Reaktion auf das Thema war: „Öhm - als Transmann schwanger zu sein (...) das geht GAR nicht das geht gar nicht«. Schwangerschaft passt für ihn nicht zu einer männlichen Identität. Er bewertet diese Phase, in der er sich eine Schwangerschaft wünschte, wie Gustav, der mutmaßt, seine Schwangerschaft sei der Versuch gewesen, sich rollenkonform zu seinem zugewiesenen Geschlecht zu verhalten. Einerseits stimmt dies sicher - der Druck auf trans Personen, sich geschlechterrollenkonform $\mathrm{zu}$ verhalten, ist enorm. Gleichzeitig scheint dies auch als Brücke zu dienen, um den Widerspruch zwischen Schwangerschaftswunsch und Unvereinbarkeit mit den eigenen Geschlechterbildern zu erklären. Kai erklärt auch, er habe durchaus Respekt vor schwangeren trans Männern wie Thomas Beatie, die offensiv mit ihrer Schwangerschaft umgingen, obwohl es für ihn gar nicht ginge. Die Ablehnung einer eigenen Schwangerschaft liegt insbesondere an der Assoziierung mit Weiblichkeit:

»Kai: Wir haben gekämpft dafür, dass wir jetzt [ja] so sind - und dann - warum sollen wir wieder etwas Weibliches an uns nehmen?, was wir ja - mit Füßen getreten haben?, [richtig] - das wär wie wenn du jetzt von mir verlangen würdest-ich soll einen Rock anziehen oder mich anfangen so zu schminken.«

Eine Schwangerschaft wird von Kai als Feminisierung empfunden, die er aufs Schärfste verhindern möchte. Seine Wortwahl, er habe das Weibliche mit Füßen getreten, zeigt, wie sehr ihn Zuschreibungen von Weiblichkeit geschmerzt haben. Gleichzeitig wird eine sehr heteronormative Vorstellung von Weiblichkeit konstruiert, in dem Frau-Sein mit Make-Up und dem Tragen von Röcken in Verbindung gebracht wird. Schwangerschaft ist für die Gruppe also mit Weiblichkeit gleichzusetzen.

Die Angst, von außen als weiblich gelesen zu werden (also vor einer Feminisierung), sowie eine internalisierte cis-normative Geschlechterordnung sorgen dafür, dass die befragten trans Männer eine Schwangerschaft für 
sich selbst ausschließen. Dies geschieht aber nicht einfach so<, sondern (wie auch der nächste Abschnitt zeigen wird) da für trans Personen das Einfügen in eine heteronormative Geschlechterordnung und Passing regelrechte Überlebensstrategien sind.

\title{
Angst vor Gewalt
}

Für die Teilnehmenden der Gruppendiskussionen sind die Themen Kinderwunsch und Schwangerschaft oft schmerzhaft, weil sie nicht ausgelebt werden können. Ein wichtiger Grund dafür ist bei den Teilnehmenden die Angst vor Gewalt. Trans Personen haben Angst, dass ihre Kinder aufgrund der trans Identität ihres Elters bzw. ihrer Eltern Gewalt erfahren und sehen es als ihre Pflicht an, ihren potentiellen Kindern dies zu ersparen.

Ein Kinderwunsch wird damit nicht nur als nicht vereinbar mit einer trans Identität verhandelt, sondern auch als verantwortungslos:

\begin{abstract}
»Mark: ähm - es könnte sein - dass ich eventuell zu dem Entschluss komme ähm - dass ich dem nicht geborenen Kinde zuliebe - weil eben die was politische oder weltliche Situation so hart ist, sage - okay ich werde diesen dies - diesen Wunsch gar nicht nachgeben weil ich - das Kind - so blöd es klingt - schon beschützen will - ohne dass es existiert ne?, weil ich könnte niemals ähm damit klarkommen, wenn meinem Kind was passiert aus dem Grund wenn ich transsexuell bin.»
\end{abstract}

Hier werden mehrere Dinge deutlich: Zum einen ist für die Diskussionsteilnehmenden trans Sein inhärent mit Gewalterfahrungen verbunden und damit, Opfer zu bringen. Zum zweiten wird Elternschaft mit Verantwortungsbewusstsein verknüpft, da sich die Teilnehmenden überlegen: »Kai: was tue ich dem Kind damit an, dass ich eben trans bin - was könnte f- für das Kind an negativen Folgen ahm kommen." Die Antwort auf diesen Konflikt ist für einige Diskussionsteilnehmer_innen, keine Kinder zu bekommen. Zum dritten werden trans Personen und ihre Körper sowie ihre Kinder über Gewalt bzw. die Androhung oder Angst vor Gewalt gesellschaftlich als normverstoßend markiert. Die Folge davon ist, dass trans Personen aus Angst vor sozialen Sanktionen ihren Kinderwunsch aufgeben. 


\section{Heteronormative Biopolitik und die Verhinderung von trans Schwangerschaften}

Es konnte gezeigt werden, dass trans Personen ihren Kinderwunsch (oftmals) nicht ausleben bzw. ausleben können und dies mit Gewalt und Diskriminierung in Medizin, Justiz und Gesellschaft zusammenhängt. Hierbei lässt sich eine Verwebung von Justiz und Medizin sehen, die, um mit Michel Foucault zu sprechen, als Biopolitik zu bezeichnen ist.

Biopolitik bezeichnet eine im 18. Jahrhundert entstehende, auf die Steigerung des Lebens ausgerichtete Machttechnologie, die über kollektive Dimensionen biologischen Lebens individuelle Körper sowie die Bevölkerung als Ganzes reguliert und normiert (Schirmer 2012:247f.; Laufenberg 2016:122; Sarasin 2005:166). Trans Schwangerschaften sind dabei nicht erwünscht, da sie Normen in Bezug auf Geschlecht, Körper, Verwandtschaft und Familie durchbrechen. Biopolitik operiert insbesondere durch die Medizin (wie ich in Bezug auf trans Schwangerschaften schon gezeigt habe) und durch die Selbstregulierung von Individuen anhand sozialer Normen (das heißt trans Personen, die sich selbst gegen eine Schwangerschaft entscheiden) (Laufenberg 2016:123).

Das bedeutet: Staatlich und medizinisch werden Schwangerschaften nur unterstützt, wenn sie in ein heteronormatives Bild passen, also die Schwangerschaften von heterosexuellen, cisgeschlechtlichen Personen in monogamen Beziehungen sind.

Während viele trans Personen einen Kinderwunsch hegen, wird dieser von den wenigsten umgesetzt. Reproduktion wird damit zu einem Sehnsuchtsthema, für das vieles, aber nicht alles, in Kauf genommen werden würde. Dabei sind meines Erachtens zwei Ergebnisse zentral: Zum einen beschreiben von mir interviewte trans Männer ihren Umgang mit einer potentiellen Schwangerschaft so, dass sie diese verstecken würden. Das queerende Potential ihrer Schwangerschaft soll dabei so wenig wie möglich sichtbar sein, da sie andernfalls Gewalt befürchten. Zum anderen zeigt sich ein hoher Pessimismus aufgrund der erlebten Diskriminierungen von trans Personen, die davon ausgehen, dass ihre Kinder Opfer von Gewalt, insbesondere Mobbing, werden würden. Somit zeigt sich, dass die psychische und physische Sicherheit, die über das Passing sichergestellt wird, deutliche Priorität vor der Erfüllung eines Kinderwunsches hat. Hiermit ist also die Erfüllung cis-heteronormativer Standards von Geschlechterbildern und Körpern ein 
wichtiges Ziel der von mir interviewten trans Personen. Allerdings erfolgt dies nicht aus einem Wunsch heraus, sondern aus der Not und erlebten Gewalt.

Eine heteronormative Biopolitik, die unter anderem darin resultiert, dass eine Vornamensänderung nach einer Schwangerschaft eines trans Manns wieder rückgängig gemacht wird, sowie Diskriminierung im medizinischen Bereich und psychische, verbale, sexualisierte und physische Gewalt durch das soziale Umfeld und der Gesellschaft als Ganzes verhindern trans Schwangerschaften. Sie lösen Angst vor einem gesellschaftlichen Unbehagen bei trans Männern aus, so dass es erst gar nicht zu einer Schwangerschaft kommt.

Fragen nach trans und Schwangerschaft sind deshalb so sensibel, weil es um Kinder geht, die immer wieder zum diskursiven Knotenpunkt werden, wenn es um Ängste, Vorurteile und den Hass cisgeschlechtlicher, heterosexueller, besorgter Eltern gegenüber queeren Personen geht. Schnell wird eine Kindeswohlgefährdung befürchtet oder vermutet, Kinder aus Regenbogenfamilien und/oder Kinder mit trans Eltern würden selbst nicht-cisgeschlechtlich und/oder nicht-heterosexuell sein. Dies ist besonders deswegen problematisch, da Familien queerer Personen generell in einer prekären rechtlichen und gesellschaftlichen Situation leben und solche Unterstellungen diese Situation noch verschärfen (können) (vgl. Janssen 2014).

\section{Neue Eugenik und heteronormative Biopolitik}

»Medical and legal discourses are part of a regulatory regime that works to eradicate the in-between spaces of gender.«(Verlinden 2012: 115) ${ }^{14}$

Im Fazit möchte ich die heteronormative, biopolitische Verhinderung von trans Schwangerschaften den Überlegungen Ute Kalenders zu neuer Eugenik gegenüberstellen. Kalender ruft dazu auf, Neuerungen in der Reproduktionsmedizin in queer_feministischen Kreisen nicht nur mit Euphorie zu begegnen, obwohl die berechtigte Hoffnung dahintersteht, dass so Menschen aller Geschlechter ihren Kinderwunsch erfüllen können, dass Geschlecht

14 Übersetzung: »Medizinische und juristische Diskurse sind Teil eines regulativen Systems, die zusammen daran arbeiten, die Zwischenräume in Bezug auf Geschlecht auszulöschen.«[AS] 
denaturalisiert wird und dass vielfältige Familien- und Verwandtschaftsformen dadurch lebbar werden. Kalender legt dar, inwiefern diese Reproduktionstechnologien mit einem Biokapitalismus zusammenhängen (Kalender 2012:198) und zeigt auf, dass in der Stammzellenforschung Eizellen von lesbischen Frauen besonders begehrt sind - womit diese zu sogenannten Rohstoffarbeiterinnen werden, ohne entlohnt oder gesetzlich abgesichert zu werden (ebd:204ff.). Es ist in diesem Zusammenhang zu erwarten, dass trans Männer ebenfalls als Gruppe markiert werden, deren Eizellen von der Reproduktionsmedizin zu Forschungszwecken begehrt werden. Zum einen aufgrund der Annahme, sie würden sie weder brauchen noch wollen und zum anderen, da die meisten Patient_innen aufgrund von Problemen bei der Befruchtung auf die Reproduktionsmedizin zurückgreifen. Allerdings haben queere cis Frauen und trans Männer als Patient_innen der Reproduktionsmedizin meistens gesunde Eizellen. Dies ist aber nicht der einzige Grund, aus dem Kalender der Reproduktionsmedizin kritisch gegenübersteht: »Die Zerstückelung«, so Kalender, »des reproduktiven Prozesses eröffnet neue Möglichkeiten für eugenische Eingriffe« (ebd. 2012:206). Die neue Eugenik zeichnet sich nicht mehr dadurch aus, dass der Staat eindeutig und direkt die Fortpflanzung von ungewünschten Personen (besonders behinderten Menschen) unterbindet. Stattdessen findet die Verhinderung von Schwangerschaften findet über gesellschaftliche Normen, Einstellungen und Ansichten statt. Es ist keine staatliche Eugenik, sondern staatliche Politik hat nur eugenische Effekte: Es pflanzen sich nur gewünschte Personengruppen fort (ebd.:207). Im Falle von trans Personen hat staatliche Politik in Form von juristischen Normen, medizinischen Behandlungsstandards und fehlender Aufklärung über trans Themen in Schulen die Folge, dass trans Personen daran gehindert werden, ihr Menschenrecht auf Familiengründung wahrzunehmen.

\section{Literatur}

Adamietz, Laura (2011): Geschlecht als Erwartung. Das Geschlechtsdiskriminierungsverbot als Recht gegen Diskriminierung wegen der sexuellen Orientierung und der Geschlechtsidentität. Baden-Baden: Nomos. Agentur der Europäischen Union für Grundrechte (FRAU, 2014): Leben als Trans in der EU. Vergleichende Datenanalyse aus der EU LGBT Erhe- 
bung. Zusammenfassung. URL: http://fra.europa.eu/sites/default/files/ fra_uploads/fra-2015-being-trans-eu-comparative-summary_de.pdf [Stand: 1.7.2018].

Beatie, Thomas (2008): Labor of Love. The Story to One Man's Extraordinary Pregnancy. Berkeley: Seal Press.

BVerfG (2011), Beschluss des Ersten Senats vom 11. Januar 2011 - 1 BvR 3295/07

- Rn. (1-77). URL: bundesverfassungsgericht.de/SharedDocs/Entschei dungen/DE/2011/o1/rs20110111_1bvr329507.html [Stand: 1.7.2018].

Cerwenka, Susanne und Nieder, Timo (2015): Transgender. In: BZgA (Hg.):

Forum Sexualität und Familienplanung 2015/1: Geschlechtsidentität und sexuelle Orientierungen. Köln, S. 17-21.

De Sutter, Petra (2014): Genetische oder biologische Trans-Elternschaft. Traum oder Wirklichkeit? In: Erik Schneider und Christel Baltes-Löhr (Hg.): Normierte Kinder. Effekte der Geschlechtsnormativität auf Kindheit und Adoleszenz. Bielefeld: transcript, S. 213-223.

Diamond, James (2011): My Pregnancy. In: Morty Diamond (Hg.): Trans/Love. Radical Sex, Love and Relationships beyond the Gender Binary. San Francisco: Manic D Press, S. 125-127.

Duhaime-Ross, Arielle (2016): Doctors Perform the First US Uterus Transplant. URL: theverge.com/2016/2/26/11119336/first-uterus-transplant-us-clevela nd-clinic [Stand: 1.7.2018].

Flütsch, Niklaus (2017): Transmenschen und Kinderwunsch. In: Gynäkologische Endokrinologie, Heft 15/1; S. 47-52.

Garfinkel, Harold (1967): Studies in Ethnomethodology. Englewood, New Jersey: Prentice Hall.

Göbel, Malte (2018): Trans* Mann gilt weiter als Mutter. URL: http://taz. de/!5515524/ [Stand: 1.7.2018].

Gordon Kaplan, Lisa (2015): Surgery Could Give Men Wombs of Their Own Within 5 Years. URL: www.yahoo.com/beauty/surgery-could-give-menwombs-1302360099545142.html [Stand: 1.7.2018].

Grady, Denise (2015): Uterus Transplants May Soon Help Some Infertile Women in the U.S. Become Pregnant. URL: www.nytimes.com/2015/11/13/ health/uterus-transplants-may-soon-help-some-infertile-women-inthe-us-become-pregnant.html?_r=1 [Stand: 1.7.2018].

Halberstam, J. Jack (2012): Gaga Feminism. Sex, Gender and the End of Normal. Boston: Beacon press. 
Hempel, Jessi (2016): My Brother's Pregnancy and the Making of a New American Family. URL: http://time.com/4475634/trans-man-pregnancy-evan/ [Stand: 1.7.2018].

Henig, Robin Marantz (2014): Transgender Men Who Become Pregnant Face Social, Health Challenges. URL: npr.org/sections/health-shots/2014/11/07 1362269036/transgender-men-who-become-pregnant-face-health-chal lenges?utm_source=facebook.com\&utm_medium=social\&utm_cam paign=npr\&utm_term=nprnews\&utm_content=202507 [Stand: 1.7.2018].

Hirschauer, Stefan (2014): Soziologie der Schwangerschaft. Explorationen pränataler Sozialität. Stuttgart: Lucius\&Lucius.

Janssen, Joke (2016): In meinem Namen. Eine trans*/queere Perspektive auf Elternschaft. In: Maya Dolderer; Hannah Holme; Claudia Jerzak und Ann-Madeleine Tietge (Hg.): O Mother where art thou? (Queer-)Feministische Perspektiven auf Mutterschaft und Mütterlichkeit. Münster: Westfälisches Dampfboot, S. 142-159.

Janssen, Joke (2014): (Mediales) Sprechen über Trans* Elternschaft. Vortrag an der Universität Hamburg. URL: https://lecture2go.uni-hamburg.de/ l2go/-/get/v/16581 [Stand: 1.7.2018].

Kalender, Ute (2012): Queere Potentiale? Zur Queerness von Reproduktionstechnologien aus der Perspektive materialistischer Feminismen und kritischer Disability Studies. Feministische Studien, Heft 30/2; S. 198-211.

Krauss, Jana (2018): WHO: Trans* Sein nicht länger »Störung von Psyche und/ oder Verhalten« URL: https://www.tgns.ch/de/2018/06/who-trans-men schen-nicht-laenger-psychisch-und-verhaltensgestoert/ [Stand:1.7.2018].

Laufenberg, Mike (2016): Die Macht der Medizin. Foucault und die soziologische Medikalisierungskritik. In: Orsolya Friedrich; Diana Aurenque; Galia Assadi und Sebastian Schleidgen (Hg.): Nietzsche, Foucault und die Medizin. Philosophische Impulse für die Medizinethik. Bielefeld: transcript, S. 109-130.

Mundlos, Christina (2015): Gewalt unter der Geburt. Der alltägliche Skandal. Marburg: Tectum.

Nordmarken, Sonny (2014): Microagressions. In: Paisley Currah und Susan Stryker (Hg.): Transgender Studies Quarterly: Postposttranssexual. Key Concepts For A Twenty-First Century Transgender Studies. Durham/ London: Duke University Press. Heft 1/2014; S. 129-133.

Obedin-Maliver, Juno und Makadon, Harvey (2016): Transgender Men and Pregnancy. In: Journal of Obsteric Medicine, Heft 9 (1); S. 4-8. 
Obedin-Maliver, Juno (2015): Time for OBGYNs to Care for People of All Genders. In: Journal of Women's Health, Heft 24/2; S. 109-111.

Pohlkamp, Ines (2015): Gender Bashing. Diskriminierung und Gewalt an den Grenzen der Zweigeschlechtlichkeit. Münster: Unrast Verlag.

Popitz, Heinrich (2010): Soziale Normen. Frankfurt a.M.: Suhrkamp.

Prantl, Heribert (2011): Das gefühlte Geschlecht. URL: www.sueddeutsche. de/politik/verfassungsgericht-kippt-transsexuellengesetz-das-gefuehl te-geschlecht-1.1052344 [Stand: 1.7.2018].

Queerulant_in (2015): Queere Politiken und Praxen. Heft 4/1.

Retzbach, Joachim (2014): Wenn Männer schwanger werden. URL: https:// www.spektrum.de/news/couvade-syndrom-wenn-maenner-schwan ger-sind/1316542 [Stand: 1.7.2018].

Richards, Annie (2016): Transsexual Pregnancy. URL: annierichards.com/ pregnant.htm [Stand: 1.7.2018].

Sarasin, Philipp (2005): Michel Foucault. Zur Einführung. Hamburg: Junius Verlag.

Schimank, Uwe (2002): Handeln und Strukturen. Einführung in die akteurstheoretische Soziologie. 2.Auflage. Weinheim/München: Juventa.

Schirmer, Uta (2012): Möglichkeiten, vergeschlechtskörpert in der Welt zu sein: Neuverhandlungen geschlechtlicher Subjektivierungsweisen im Kontext des medizinsch-rechtlichen Regimes der Transsexualität. In: Eva Sänger und Malaika Rödel (Hg): Biopolitik und Geschlecht. Zur Regulierung des Lebendigen. Münster: Westfälisches Dampfboot, S. 244265.

Spahn, Annika (2017): Subversion oder Assimilation? Trans und Schwangerschaft in einer heteronormativen Gesellschaft. URL: https://freidok. uni-freiburg.de/data/12314 [Stand: 1.7.2018].

Toze, Michael (2018): The Risky Womb and the Unthinkability of the Pregnant Man: Adressing Trans Masculine Hysterectomy. In: Feminism \& Psychology, Heft 28/2; S. 194-211.

Valentine, David (2007): Imagining Transgender. An Ethnography of a Category. Durham/London: Duke University Press.

Verlinden, Jasper (2012): Transgender Bodies and Male Pregnancy: The Ethics of Radical Self-Refashioning. In: Michaela Hampf und MaryAnn Snyder-Körber (Hg.): Machine: Bodies, Genders, Technologies. Heidelberg: Universitätsverlag Winter, S. 107-136. 
Villa, Paula-Irene/Moebius, Stephan und Thiessen, Barbara (2011): Soziologie der Geburt: Diskurse, Praktiken und Perspektiven. Einführung. In: dies. (Hg.): Soziologie der Geburt: Diskurse, Praktiken und Perspektiven. Frankfurt a.M.: Campus Verlag, S. 7-21.

Wallace, J. (2010): The Manly Art of Pregnancy. In: Kate Bornstein und Bear Bergman (Hg.): Gender Outlaws. The Next Generation. Berkeley: Seal Press, S. 188-194.

Wierckx, Katrien; Van Caenegem, Eva; Pennings, Guido; Elaut, Els; Dedecker, David; Van de Peer, Fleur; Weyers, Steven; De Sutter, Petra und T'Sjoen, Guy (2012): Reproductive Wish in Transsexual Men. In: Human Reproduction, Heft 27/2; S. 483-487. 
